Pramana
$\begin{array}{r}\text { journal of } \\ \text { physics }\end{array}$

TIFR/TH/14-13

\title{
QCD Critical Point: The Race is On
}

\author{
RAJIV V. GAVAI ${ }^{\mathrm{a}, *}$ \\ ${ }^{a}$ Department of Theoretical Physics, Tata Institute of Fundamental Research, \\ Homi Bhabha Road, Mumbai 400005, India.
}

\begin{abstract}
.
A critical point in the phase diagram of Quantum Chromodynamics (QCD), if established either theoretically or experimentally, would be as profound a discovery as the good-old gas-liquid critical point. Unlike the latter, however, first-principles based approaches are being employed to locate it theoretically. Due to the short lived nature of the concerned phases, novel experimental techniques are needed to search for it. The Relativistic Heavy Ion Collider (RHIC) in USA has an experimental program to do so. This short review is an attempt to provide a glimpse of the race between the theorists and the experimentalists as well as that of the synergy between them.
\end{abstract}

Keywords. Critical Point, Quantum Chromodynamics, Heavy Ions Collisions, Lattice QCD

PACS Nos. 12.38.Gc, 11.35.Ha, 05.70.Jk

\section{Introduction}

Critical points in a phase diagram in the temperature-density plane are special for several reasons. Starting from their rarity, only one or two in usual systems, to the various dramatic changes in a host of physical quantities at the critical point are the observationsbased curiosities, while theoretically the universality of critical indices, diverging correlation length etc. enable us to relate widely different physical systems to the common underlying symmetries. For common substances, such as water or carbon dioxide, the existence of critical point has been established experimentally, with its location known rather precisely. Critical exponents for magnetic systems have been found experimentally to be equal to those for the many liquid-gas systems, and are related to the symmetry of the Ising model. For many of the physical systems, however, getting a theoretical, especially first principles based, computation of their locations has turned out to be illusive still. The aim of this article is to describe how things are different for strongly interacting matter, which is naturally described by Quantum Chromo Dynamics (QCD). QCD has an inherently a higher energy, and thus a shorter time, scale. Whether the QCD phase diagram has a critical point in its temperature $T$ and the baryonic chemical potential $\mu_{B}$ plane is therefore a tougher question to address experimentally as well. Thanks to the impressive developments on the experimental fronts, the Relativistic Heavy Ion Collider (RHIC) at BNL, New York, and the upcoming facilities such as FAIR at GSI, Darmstadt and NICA at Dubna, Russia may be able to search for it, and potentially even locate it.

*gavaietifr.res.in 
Unlike its cousin QED -quantum electrodynamics- QCD turns out to be much richer in structure. While it can be handled theoretically in the (almost) same way as QED when the coupling between its constituents quarks and gluon is small, newer tools are needed in the strong coupling regime where a lot of the rich structure, such as quark confinement, exists. A variety of models have been developed to handle this domain, and have been tested successfully for hadronic interactions to a limited extent. These models were was also the first set of tools used for getting a glimpse of the QCD phase diagram [1]. For instance, using an effective chiral Nambu-Jana Lasinio type model, a phase diagram was obtained, which suggests [2] a critical point to exist in a world with two light quarks (up and down flavours) and one moderately heavier (strange) quark. One would clearly like to have an ab initio theoretical evidence for it from QCD directly. This turns out to be difficult as one has to deal with the strong coupling regime of QCD. Nonperturbative Lattice QCD, defined on a discrete space-time lattice, has proved itself to be the most reliable technique for extracting the information from QCD in the world of low energy hadronic interactions. The hadron spectrum has been computed and predictions of weak decay constants of heavy mesons have been made [3]. Numerical simulations of lattice QCD using the importance sampling approach have been the backbone for all such computations. Since use of these techniques was actually inspired by their success in statistical mechanics, it is natural to extend the same approach to finite temperature and finite density QCD. Indeed, its application to finite temperature QCD has also yielded a slew of results for QCD thermodynamical observables, such as the pressure as a function of temperature. It is therefore natural to ask whether lattice QCD can help us in locating the QCD critical point. The prospect of finding the critical point experimentally makes it exciting both as a check of such theoretical predictions and as a competition for getting there first. Clearly, in view of the complexity of the task, one could turn to lattice QCD again to see if it can provide any hints for the experimental search program as well. In this short review, I aim to summarise the results obtained in this direction, including those by the TIFR group, Mumbai.

Lattice QCD simulations at finite density, or equivalently nonzero $\mu_{B}$, have had to face to two severe problems, which have resulted in a slow progress in the field of QCD critical point. Recently, one of them, a conceptual issue, appears to have been solved. Let me explain that first. Due to the well-known fermion doubling problem, one has to make a compromise in choosing the quark type for any lattice QCD computation. While the final results should be independent of the quark type, indeed any form of the lattice action, the choice of the quark type is often dictated by the nature of the task at hand. The popular choice in finite temperature and density studies has been mostly the Kogut-Susskind (staggered) quarks [4]. They have an exact chiral symmetry at any value of the lattice spacing (any finite cut-off) which leads to an order parameter, the chiral condensate $\langle\bar{\psi} \psi\rangle$, for a lattice investigation in the entire $T-\mu_{B}$ plane. Unfortunately though, the flavour and spin symmetry is broken for them on a lattice. A simulation relevant to experiments ought to have 2 light (or 2 light +1 heavier ) quark flavours. Precisely such a representation for staggered quarks may not be feasible, more so on the coarse lattices one is constrained to employ. The existence of the critical point, on the other hand, is expected to depend crucially on the number of flavours. Flavour and spin symmetry is therefore needed in simulations. Although computationally much more expensive, domain wall or overlap fermions [5] are better in this regard, as they do have the correct symmetries for any lattice spacing at zero temperature and density. Introduction of chemical potential, $\mu$, for these is, however, not straight-forward due to their non-locality. Bloch and Wettig [6] proposed a way to do this. Unfortunately, it turns out [7] that their prescription breaks chiral 
QCD Critical Point: The Race is On

symmetry. Of course, a space-time lattice breaks even translational and rotational symmetry. Exactly as these are expected to be restored in the continuum limit of vanishing lattice spacing, one may argue that the same would be the case for the Bloch-Wettig method. The problem is that for the chiral condensate to be an order parameter on a lattice, the chiral symmetry must be respected even on the lattice. Otherwise cut-off dependent corrections to it would change it in an uncontrollable way. Furthermore, the chiral anomaly for the Bloch-Wettig action depends on $\mu$ unlike in continuum QCD [8]. As a result, it too may alter the existence/location of the critical point on the lattice. Recently, this conceptual issue has been resolved [9] by a new proposal to introduce the chemical potential for both overlap and domain wall fermions. It leads to a formalism with both a) continuum-like (chiral, flavour and spin) symmetries for quarks at nonzero $\mu$ and $T$ and b) a well-defined order parameter on the lattice.

Finite density simulations needed for locating a critical point suffer from another well known problem. It is inherited from the continuum theory itself: the fermion sign problem. It appears in areas of statistical mechanics also and has so far remained unsolved. It is a major stumbling block in extending the lattice techniques to the entire $T-\mu_{B}$ plane. Simply stated, simulations rely on importance sampling due to the large number of field (quark and gluon) variables. Assuming $N_{f}$ flavours of quarks, and denoting by $\mu_{f}$ the corresponding chemical potentials, the QCD partition function is

$$
\mathcal{Z}=\int D U \exp \left(-S_{G}\right) \Pi_{f} \operatorname{Det} M\left(m_{f}, \mu_{f}\right),
$$

and the thermal expectation value of any observable $\mathcal{O}$ is

$$
\langle\mathcal{O}\rangle=\frac{\int D U \exp \left(-S_{G}\right) \mathcal{O} \prod_{f} \operatorname{Det} M\left(m_{f}, \mu_{f}\right)}{\mathcal{Z}} .
$$

For a $N_{s}^{3} \times N_{t}$ lattice, the simulations then correspond to a spatial volume of $V=$ $N_{s}^{3} a^{3}$, and a temperature of $T=\left(N_{t} a\right)^{-1}$, where $a$ is the lattice spacing. Quark masses and chemical potentials are then also in units of $a$. Typically, the integral above is 10 million dimensional and $M$, the fermion matrix defined by the lattice Dirac operator, is about a million $\times$ million. Probabilistic methods are therefore used to evaluate $\langle\mathcal{O}\rangle$. These need a positive definite measure. From Eq.(1), one sees the measure to be proportional to the exponential of the gluonic action, $\exp \left(-S_{G}\right)$ and the fermionic determinant Det $M$. While the former factor is positive definite, for nonzero $\mu$, the determinant turns out to be a complex number, although the partition function itself remains real. This is the fermion phase/sign problem in finite density QCD. Note that the determinant is purely real for $\mu=0$. Several approaches have been proposed in the past to deal with it. In view of the space constraints, let me provide a partial list, with some comments:

1. Suitable choice of variables $[10,11]$ - Since the partition is still real, one can attempt suitable variable transformations to re-write it using variables which do not lead to a complex measure. The key here is, of course, to find such variables. This approach has been shown to work in simpler models or with some drastic approximations to QCD but so far remains a distant goal for realistic QCD simulations.

2. Imaginary chemical potential [12] - Substituting $\mu \rightarrow i \mu$ in the fermion determinant, one can easily verify that it becomes real. Usual simulation methods then enable one to explore the imaginary $\mu$-axis. However, the results so obtained can 
only be employed for our world after analytic continuation, which introduces unknown and uncontrollable errors. So far, it has been used for very coarse $\left(N_{t}=4\right)$ and with only the leading term in an polynomial ansatz for analytic continuation. No hints of QCD critical point were seen. It will be interesting to see how these results will be in the continuum limit and with more terms in the polynomial.

3. Canonical ensemble method [13] - Computations at a fixed baryon number do not have the fermion sign problem as well. Using this idea, simulations on small lattices have been done with encouraging results. One needs to compute them in the limit of very large baryon number in order to obtain the corresponding chemical potential. Until that is achieved, one can only make qualitative comparisons. The hints of a first order phase transition seen in these simulations are consistent with a possible critical point at some other, perhaps lower, $\mu$.

4. Complex Langevin approach [14] - Stochastic quantization of field theories aims to evaluate the $\langle\mathcal{O}\rangle$ by formulating the problem in terms of a Langevin equation and by employing its (simulation) time evolution. It too, of course, needs the same reality and positivity condition. Although, a formal proof does not exist for complex action, one may still hope that it converges to the correct answer. Such attempts for some models have been shown to work, i.e., they agree with the known exact/semianalytic results but in some cases it is known to fail as well. Currently attempts are being to made to understand this to see if it can be eventually applied to full QCD.

5. Two parameter re-weighting [15] - Importance sampling, basic to many of the simulation methods, works by creating the most probable distribution of the action in Eq. (1) for given input parameters, such as the gauge coupling. Exploiting cleverly the fact that such a distribution can be constructed for $\mu=0$ by using standard methods, this approach consists of obtaining the desired $\mu \neq 0$ distribution from the expectation values with respect to the former. Fodor and Katz [15] were the first to obtain a lattice result for the QCD critical point on small and coarse lattices $\left(4^{3}-8^{3} \times 4\right)$, which was found to be at $\mu_{E} / m_{N u c l e o n} \sim 0.77$. Employing similar lattice but a realistic quark mass spectrum in [15], they obtained a critical point at a factor 2 lesser $\mu_{E}$. Extending this approach to larger and fine lattices appears not to be easy because of an exponential growth in the size of the computational resources needed due to the so-called 'overlap' problem but would be nice if it could be done.

6. Taylor expansion [16, 17] - The Taylor expansion approach was developed by the TIFR group [16] and the Bielefeld group [17]. Results obtained by this method have been rather encouraging, as one has been able to study systematically i) the finite volume effects to go towards a thermodynamical limit $\left(N_{t} \rightarrow \infty\right)$ and ii) the continuum limit of vanishing lattice spacing $\left(N_{t} \rightarrow \infty\right.$ or equivalently $\left.a \rightarrow 0\right)$. I will discuss some details of these results in the next section.

\section{Lattice Results}

Expanding the partition function of Eq. (1) near $\mu_{f}=0$, where $f$ is the flavour index denoting up, down, strange..quarks, one can evaluate the coefficients of the various terms by employing the usual simulation method since they are evaluated for $\mu_{f}=0$ for which the determinant is real. Moreover, both the thermodynamic limit, $N_{s} \rightarrow \infty$, and the continuum limit, $N_{t} \rightarrow \infty$ of these coefficients can be evaluated in the same way as the hadron spectrum can be computed in these limits to obtain the continuum results. We will 
consider below only two flavours, up and down, with the corresponding chemical potentials, $\mu_{u}$ and $\mu_{d}$ for simplicity. Generalizing to any number of flavours is straightforward, although for our world two light and one moderately heavy flavour may suffice.

Using standard definitions, number densities $n_{i}$ and the corresponding susceptibilities $\chi_{i j}$ can be obtained from Eq. (1) as respectively the first and second derivatives of the partition function $\mathcal{Z}$ :

$$
n_{i}=\left.\frac{T}{V} \frac{\partial \ln \mathcal{Z}}{\partial \mu_{i}}\right|_{T=\text { fixed }} ; \quad \chi_{i j}=\left.\frac{T}{V} \frac{\partial^{2} \ln \mathcal{Z}}{\partial \mu_{i} \partial \mu_{j}}\right|_{T=\text { fixed }} .
$$

Natural units with the Boltzmann constant $k$ set to unity have been used above. While the $n_{i}$ are zero for $\mu_{i}=0$, the susceptibilities are a nontrivial function of temperature. Indeed, these are known to play many roles in the physics of finite temperature phase transition, including acting as independent indicators of the transition itself. Denoting by $\chi_{n_{u}, n_{d}}$ higher derivatives of $\ln \mathcal{Z}$ at the $n_{u}^{\text {th }}\left(n_{d}^{\text {th }}\right)$ order with respect to $\mu_{u}\left(\mu_{d}\right)$, the QCD pressure $P=T \ln \mathcal{Z} / V$ is seen to have the following expansion in $\mu_{i}$ :

$$
\frac{\Delta P}{T^{4}} \equiv \frac{P(\mu, T)}{T^{4}}-\frac{P(0, T)}{T^{4}}=\sum_{n_{u}, n_{d}} \chi_{n_{u}, n_{d}} \frac{1}{n_{u} !}\left(\frac{\mu_{u}}{T}\right)^{n_{u}} \frac{1}{n_{d} !}\left(\frac{\mu_{d}}{T}\right)^{n_{d}}
$$

At a generic point $(\mu, T)$ in the QCD phase diagram, one should be able to to sum the series for the pressure as a function of temperature $T$. In presence of a critical point at $T_{E}$, however, a finite radius of convergence in $\mu$ would limit the range up to which such a summation can be done. A typical lattice computation can thus aim to search for the QCD critical point by looking for the radius of convergence of the series at several temperatures. Since the baryonic susceptibility should have a stronger power law divergence at the critical point, it was proposed [18] to use that series instead of that of pressure above. It is easy to construct the series for baryonic susceptibility from the above expansion and look for its radius of convergence as the estimate of the nearest critical point.

Due to the symmetry between baryons and antibaryons at $\mu=0$, odd coefficients of the series vanish. Using the even ones, successive estimates for the radius of convergence can be obtained by using the usual ratio method

$$
r_{n+1, n+3}=\sqrt{\frac{n(n+1) \chi_{B}^{(n+1)}}{\chi_{B}^{(n+3)} T^{2}}}
$$

and the $\mathrm{n}^{\text {th }}$ root method

$$
r_{n}=\left(\frac{n ! \chi_{B}^{(2)}}{\chi_{B}^{(n+2)} T^{n}}\right)^{1 / n} .
$$

Of course, one needs to evaluate the $n \rightarrow \infty$ limit of the radii estimates above to arrive at the true radius of convergence. Due to the computational complexity involved, both the number of terms and cancellations amongst these terms increase as $n$ grows, terms up to 8th order in $\mu$ have been used so far [18]. A proposal [8] to alleviate these problems, and thus get more coefficients for the same computational resources has been made. It consists of adding the chemical potential simply as $\mu N[8]$, in contrast the commonly used form 
exponential in $\mu$. The linearity in $\mu$ implies that most derivatives of the quark matrix with $\mu$ are zero except the first one. This reduces a lot the number of terms in each nonlinear susceptibility (NLS) appearing in the equations above. One can show that it still leads to essentially the same results[20]. It would be interesting to see the results for the radius of convergence obtained by using it.

Since the QCD critical point is expected to occur for real $\mu$, a key point to note is that all the coefficients of the series must be positive. Having made sure that it is so, one can then look for agreement between the two definitions above as well as their $n$-independence to locate the critical point. The detailed expressions for all the terms can be found in [18] where the stochastic estimators method to evaluate them is also explained. For terms up to the $8^{t h}$ order one needs 20 inversions of the Dirac matrix, $(D+m)$, on $\sim 2000$ vectors for a single measurement on a given gauge configuration. At least hundreds of such measurements are necessary to obtain the results discussed below; more precise results will need even more. This makes the computation very time consuming. Nevertheless, extension to 10 th $\&$ even 12 th order may be possible, using the idea in [8] which may save up to $60 \%$ computer time in these measurements.

The results of Ref. [18, 19, 21] were obtained by simulating full QCD with two flavours of staggered fermions of mass $m / T_{c}=0.1$ on $N_{t} \times N_{s}^{3}$ lattices, with $N_{t}=4$ and $N_{s}=8,10,12,16,24$ [18], a finer $N_{t}=6$ with $N_{s}=12,18,24$ [19] and a still finer $N_{t}=8$ and $N_{s}=32$ lattice. Here $T_{c}$ denotes the transition temperature at $\mu=0$. It is obtained by studying the point of inflection in variables like the chiral order parameter or the Polyakov line. Earlier work by the MILC collaboration for the smaller $N_{t}=$ 4 lattice had determined [22] the ratio of the mass of the rho particle to the transition temperature, $m_{\rho} / T_{c}=5.4 \pm 0.2$ and the similar ratio for pion, $m_{\pi} / m_{\rho}=0.31 \pm 0.01$. This amounts to a Goldstone pion of $230 \mathrm{MeV}$ in that case, which is heavier than the physical pion mass of $140 \mathrm{MeV}$. For the finer lattices, Ref. [19, 21] had to determine the $\mu=0$ transition points $\beta_{c}$ as well. It was checked that these were consistent with the nearby quark mass results in the literature. Covering typically a temperature range of $0.89 \leq T / T_{c} \leq 1.92$ by suitably choosing the range of couplings on all lattices, the coefficients were determined typically on 50-200 independent configurations, separated by the respective autocorrelation lengths.
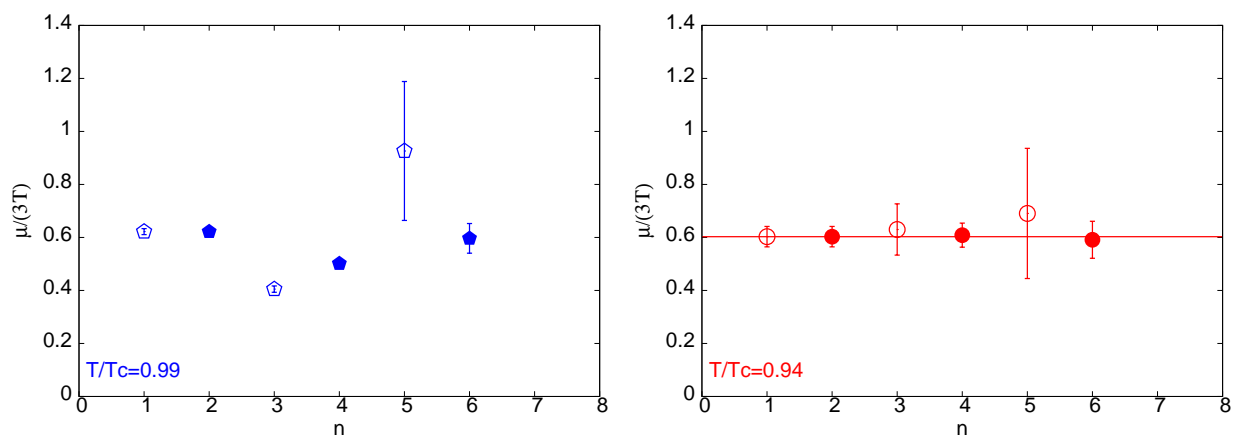

Figure 1. Estimates of radii of convergence as a function of the order $n$ and for the two methods (see text) at $T / T c=0.99$ and the critical point $T^{E} / T_{c}=0.94$. The open (filled) points display the results for the ratio (root) method. From Ref. [19].

Fig. 11 shows the results [19] for both the ratios defined above on the $N_{t}=6$ lattice at two different temperatures, $T / T_{c}=0.99$ and 0.94 . In order to exhibit both the results of eqs. (5 6) the $n$ on the $\mathrm{x}$-axis was suitably transformed in those equations such that the 
hollow (filled) symbols show the ratios $\left(n^{\text {th }}\right.$ root). All the susceptibilities are positive at both the temperatures but the ratios fluctuate for the former temperature while seeming to be constant for the latter, indicating it to be the critical temperature and their constant value to be the $\mu_{u} / T=\mu_{d} / T=\mu_{B} / 3 T$. Ref. [19] thus found the coordinates of the endpoint (E)—the critical point- to be $T^{E} / T_{c}=0.94 \pm 0.01$, and $\mu_{B}^{E} / T^{E}=1.8 \pm 0.1$.

As a cross check on the location of the $\mu^{E} / T^{E}$, one can use the series with the same numerically determined coefficients to construct $\chi_{B}$ for nonzero $\mu$ directly. As one sees in the left panel of Fig. 2, this leads to smooth curves with no signs of criticality irrespective of how many terms one uses to determine the curve. This is, of course, to be expected, since the series is merely a finite (and small) order polynomial. It is well known from statistical mechanics that Padé approximants are a better guide in such cases. Employing the Padé approximants for the same series to estimate the radius of convergence does lead to a consistent window with the above estimate, as seen the right panel of Fig. 2
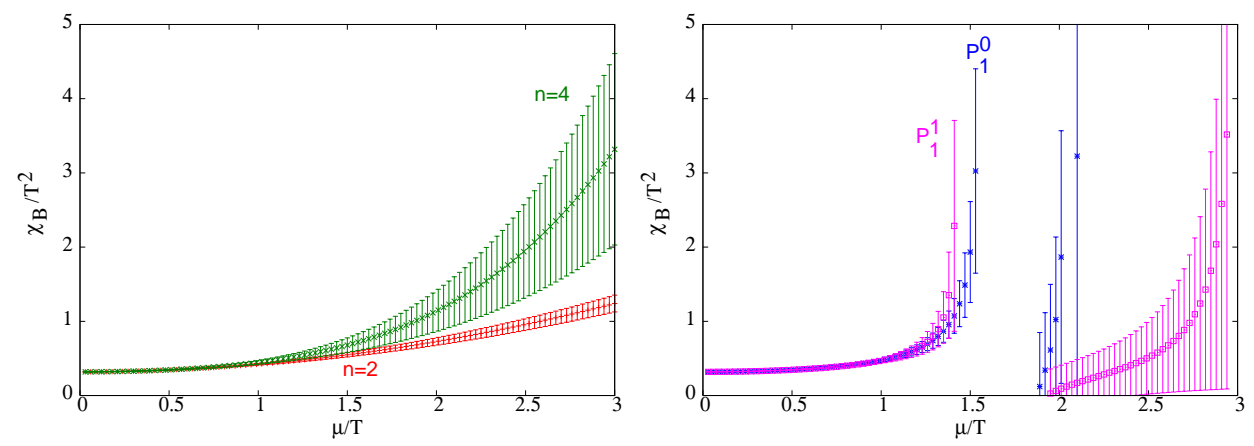

Figure 2. The baryonic susceptibility as a function of the baryonic chemical potential using simple polynomial with all computed orders (left) and Padé approximants (right). From Ref. [19].

The left panel of the Fig. 3 displays a comparison of the results on the even finer $N_{t}=8$ lattice [21] with those for the $N_{t}=6$ lattice. Significantly more computation power is needed for the larger lattice not only because the computations grow as $N_{s}^{3} \times N_{t}$ but also cancellations of various terms become more delicate. As in the cases of the smaller $N_{t}=4$ and 6 lattices, the data for the appropriate radii-estimates for $\mu_{E} / T_{E}$ exhibit near constancy for the various possible ratios of the Taylor coefficients. The first (last) three data point display the $n^{\text {th }}$ root (ratio) results. The two bands are the final estimates for $N_{t}=6$ (cyan) and 8 (green) lattices [19, 21]. One sees that they agree within errors whereas the earlier coarsest [18] lattice result was at a somewhat smaller $\mu_{B} / T$. The right panel of Fig. 3 shows the QCD phase diagram with known lattice determinations $[15,18,19,21]$. Given the complexity of the problem it is very encouraging that a) two different methods with their own different shortcomings and 2) three different $N_{t}$ or equivalently three different cut-offs lead to nearby determinations of the location of the critical point. The agreement within error bands for the two finest lattices is further encouraging. One has to bear in mind though that there is still quite a long way to go. Cut-off independence (still larger $N_{t}$ ), volume independence (larger aspect ratio $N_{s} / N_{t}$ than 4 employed here) as well as the independence from other choices, such as the type of quarks or method have to be further established. The simulations also need to come closer to the continuum QCD by having physical pion mass, i.e., lower dynamical quark mass, and the correct $U_{A}(1)$ anomaly. Nevertheless, one can hope that the concurrence of these results can already motivate strong efforts to locate the critical point experimentally, 
and as we shall see below, even provide some guidance on how to decipher that from the hadronic data visible to the detectors.
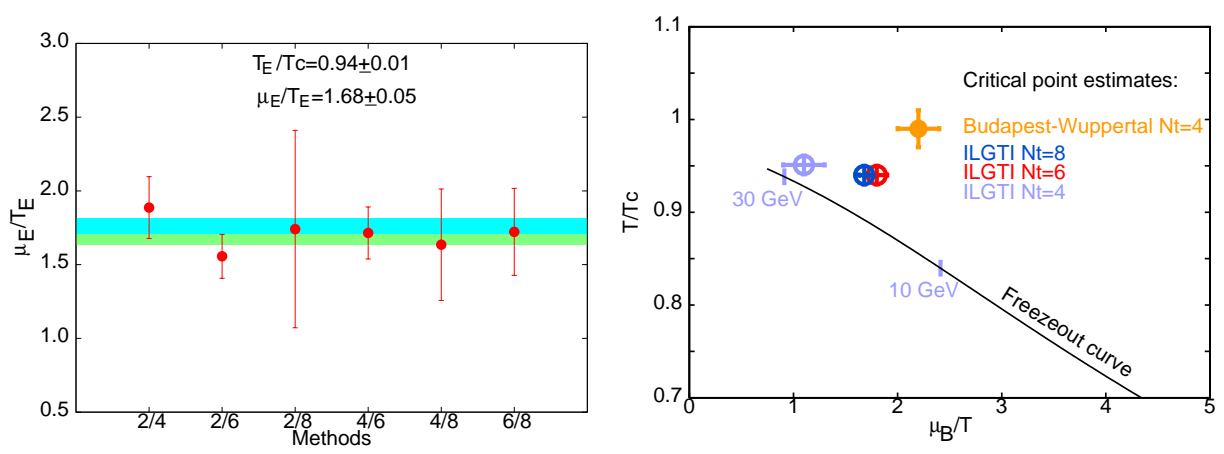

Figure 3. Estimates of radii of convergence as a function of the ratios used for the two methods at the critical point $T^{E} / T_{c}$ for $N_{t}=8$ (data) along with the final results [21] for $N_{t}=8$ (green band) and 6 (cyan band) lattices. The right panel shows the QCD phase diagram from known lattice determinations [15, 18, 19, 21]. See text for details.

\section{Searching Experimentally}

Searching for the QCD critical point experimentally also entails several formidable challenges, in view of the anticipated high values of its temperature and density. Bjorken [23] argued that matter at such temperatures may be created in relativistic heavy ion collisions. Indeed, major experimental efforts at BNL, New York and CERN, Geneva in the past few decades have yielded spectacular results [24] supporting such an idea. It was observed that huge amounts of precise data on hadron abundances in a range of relativistic heavy ion experiments at several colliding energies $(\sqrt{s})$ are well described [25] by statistical thermodynamical models with minimal parameters such as temperature $T$ and the baryonic chemical potential $\mu_{B}$. It led to a mapping of the $T$ and $\mu_{B}$ parameters of the model to the collision (center of mass) energy $\sqrt{s}$, the so-called freeze-out curve. One such parameterization of the freeze-out curve is shown in the QCD phase diagram in the right panel of Fig. 3. Although, this curve can, on one hand, be deemed as a mere parameterization of the hadron yields in the heavy ion collision experiments, it has the advantage of being solely obtained from precise experimental data and provides the $(T, \mu)$ accessible in these heavy-ion experiments as a function of the colliding energy $\sqrt{s}$. Identifying them as the thermodynamic variables of the fireball created therefore gives us an idea of the likely colliding energy one may need to employ to explore the region where the QCD critical point may be expected. One expects it to be at rather small colliding energies than the original RHIC design of $200 \mathrm{GeV}$ or the LHC energy. The encouraging aspect of the lattice results in Fig. 3 is that the expected range of $\sqrt{s}$ is not too low, as would be the case if the critical point were to be at too high a $\mu_{B}$. It turned out that the RHIC accelerator could be tuned to run at lower $\sqrt{s}$, a technologically impressive feat since it was not designed for that. Considering the spread in the values of lattice $\mu_{E} / T$ in Fig. 3. a beam energy scan program was proposed and sanctioned. I shall mostly concentrate below on some of the results of these program. For more details, I refer the reader to the experimental review in this volume.

Since the well-known experiments of Thomas Andrews in 1869 for the liquid-gas transition in carbon dioxide, one expects critical opalescence, or huge fluctuations, to char- 
QCD Critical Point: The Race is On

acterise the second order phase transition at the critical point. A diverging correlation length $\xi$ is now understood to be the cause for this phenomenon, and is related to the diverging specific heat or susceptibility at the critical point. Stephanov, Rajagopal and Shuryak [28] proposed that studying the event-by-event fluctuations of suitably chosen observables in heavy ion collisions as a function of $\sqrt{s}$ ) and suitable kinematic cuts can be used to search for the QCD critical point due to the nonmonotonicity a critical point should induce in them. They proposed to focus on the fluctuations, or the second moments, of the observables constructed from the multiplicity and transverse momenta of charged pions. These have been investigated with no conclusive results. Recognising that the higher moments of the same variables grow as a significantly higher power of $\xi$, Stephanov [29] suggested fourth and higher moments to be a more attractive tool.

Considering that the QCD critical point arises due to a variation in the baryon density, such fluctuations in baryon number, given by the appropriate combinations of the many quark number susceptibilities discussed in the previous section, appear a more natural choice [30, 31]. In order to make predictions for the heavy ion experiments, and design further any search criteria for the critical point, it was proposed [31] to use the freeze-out curve as a a way to set the temperature $T$ and the chemical potential $\mu_{B}$ in the lattice QCD computations to compute the various fluctuations. The information hidden in the nonlinear susceptibilities discussed in the previous section can then be extracted by evaluating lattice QCD predictions along it. Not only does such an approach amounts to assuming that the $(T, \mu)$ along the freeze-out curve do reflect the true thermodynamic variables of the system, but it also takes the model [25] a step further and aims to predict the fluctuations in the hadron abundances from first principles. Comparing these lattice predictions with data even at large $\sqrt{s}$, away from the critical point, thus is tantamount to a non-perturbative test of QCD, subject to the assumptions outlined already.

One expects the QCD critical point to have a critical region whose size depends on the size of the fireball as well as its critical exponents. The freeze-out curve may pass through it or may miss it. In the former case, one should expect the fluctuations to be enhanced in the experiments but nothing spectacular may happen in the latter case. If the conditions are right, it may even pass close enough to the critical point such that a study of fluctuations along it will detect its presence unambiguously. Defining $m_{1}=$ $T \chi^{(3)}\left(T, \mu_{B}\right) / \chi^{(2)}\left(T, \mu_{B}\right), m_{3}=T \chi^{(4)}\left(T, \mu_{B}\right) / \chi^{(3)}\left(T, \mu_{B}\right)$, and $m_{2}=m_{1} m_{3}$, and noting that the variance, skewness and kurtosis of the event distribution of baryon number measure the various $\chi$ 's appearing in them, one sees that these ratios can be computed from lattice studies as well as from the experimental data. They are designed [30, 31] such that the spatial volume cancels out in them, making them suitable for both i) lattice studies, which can potentially have finite volume effects, and ii) experiments which too are unable to pin down the volume of the fireball and may prefer to use their favourite proxy for it. Usually, number of participants is preferred for that role in the analysis of heavy ion data. All lattice results also have to worry about the finite lattice cut-off effect; one has to take the cut-off away (lattice spacing $a \rightarrow 0$ or $N_{t} \rightarrow \infty$ ) by some extrapolation to obtain continuum results. Since dimensionless ratios have generally found to vary rather slowly with $a$ for small enough $a$, the dimensionless $m_{i}$ 's are also expected to be reasonably cut-off independent.

Defining $z=\mu_{B} / T$, and denoting by $r_{i j}$ the estimate for radius of convergence using $\chi_{i}, \chi_{j}$ as before, one has

$$
m_{1}=\frac{2 z}{r_{24}^{2}}\left[1+\left(\frac{2 r_{24}^{2}}{r_{46}^{2}}-1\right) z^{2}+\left(\frac{3 r_{24}^{2}}{r_{46}^{2} r_{68}^{2}}-\frac{3 r_{24}^{2}}{r_{46}^{2}}+1\right) z^{4}+\mathcal{O}\left(z^{6}\right)\right]
$$


Note that the coefficients of the polynomial above are determined by the zero density $(\mu=0)$ simulations as described in the previous section and depend only on temperature. Similar series expressions can be written down for $m_{2}$ and $m_{3}$ as well. In order to capture the critical behaviour, where at some $T_{E}$ all these radii tend to be equal, a simple series summation may be inadequate, as in Fig. 2. One should resum these by the Padè method as above to construct $m_{i}(\sqrt{s}) \equiv m_{i}\left(z=\mu_{B} / T, T\right)$, since $)$ the Padè approximants seem to capture the critical behaviour well in Fig. 2, and ii) computations along the freeze-out curve guarantee the equivalence for $m_{i}$. A suitable ansatz is :

$$
m_{1}=z P_{1}^{1}\left(z^{2} ; a, b\right), \quad m_{3}=\frac{1}{z} P_{1}^{1}\left(z^{2} ; a^{\prime}, b^{\prime}\right)
$$
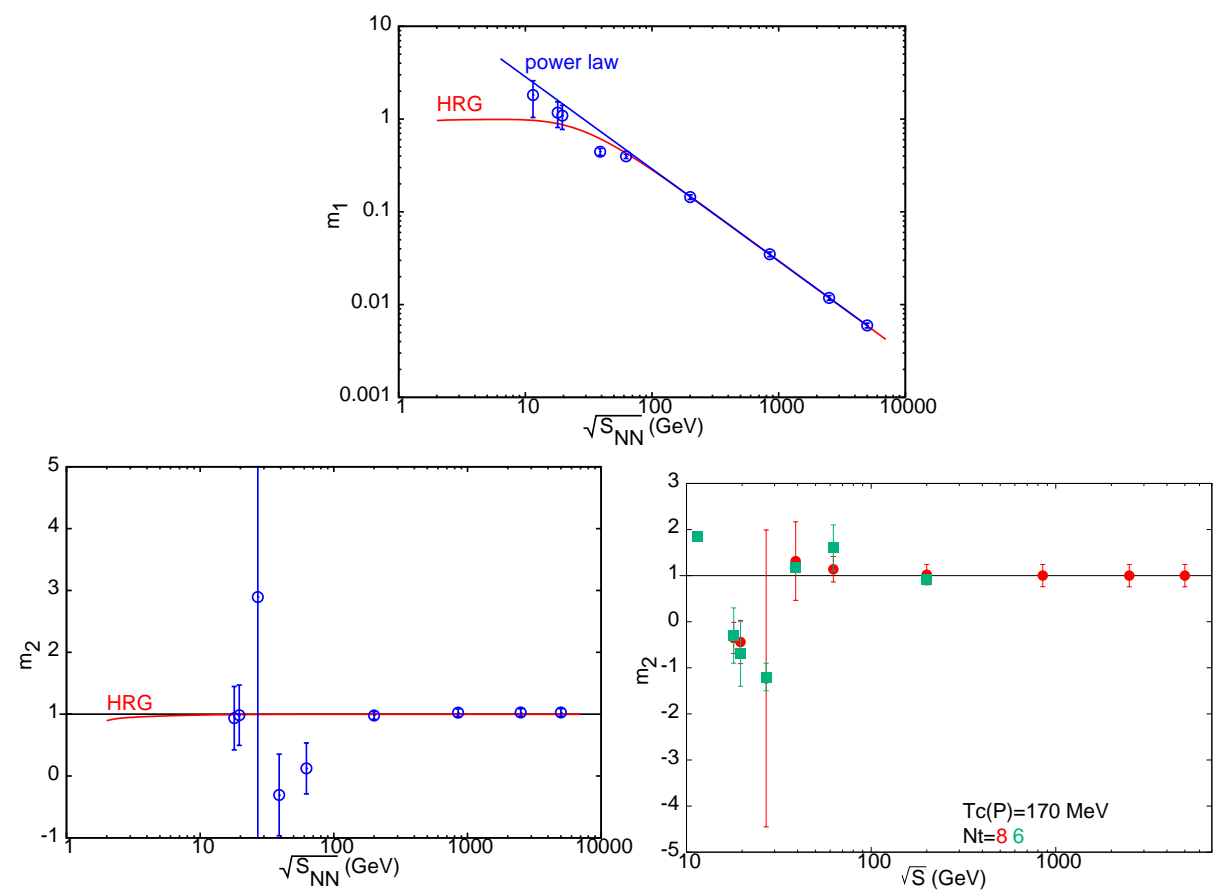

Figure 4. Results for $m_{1}$ (top) and $m_{2}$ (bottom left) on a $N_{t}=6$ lattice as a function of the colliding energy. A power law fit to the high energy data and a hadron resonance gas fit is also shown. The bottom right panel shows preliminary results for $m_{2}$ on the $N_{t}=8$ (green) along with the $N_{t}=6$ (red) lattices. From Ref. [31, 32].

The top panel and the bottom left panel in Figure 4 display the results [31] for the ratios $m_{1}$ and $m_{2}$ on the $N_{t}=6$ lattice; the results for coarser $N_{t}=4$ lattice are similar and can be found in Ref. [31]. Also shown in both these figures are results for a hadron resonance gas which does not assume the existence of a critical point. The bottom right panel compares the preliminary results for $m_{2}$ for the $N_{t}=8$ [32] lattices along with those for the $N_{t}=6$. A general agreement in it is an indication again of lattice cut-off effects being rather small. The source of the large errors at the critical point for $N_{t}=8$ could very well be the critical fluctuations themselves. One needs to investigate this by varying the statistics of the gauge configurations as well as the number of vectors employed to estimate each quark propagator. In all the figures, one observes 
QCD Critical Point: The Race is On

a smooth and monotonic behaviour for large $\sqrt{s}$ which is well reproduced by the hadron resonance gas. Note that even in this smooth region, any experimental comparison is exciting since it is a direct non-perturbative test of QCD in hot and dense environment, subject to the assumption that the freeze-out curve $T$ and $\mu_{B}$ actually do correspond to those of the fireball produced in those experiments. Other lattice QCD predictions, such as the equation of state or the transition temperature can be compared with experiments only indirectly by employing them in a hydrodynamical simulation and comparing the simulation results, e.g., for the elliptic flow, with the corresponding experimental results. Since initial conditions in such hydrodynamical simulations are not uniquely known, such an indirect comparison hinges on several other modelling aspects.

Remarkably, a non-monotonic behaviour is visible in Fig. 4 at the estimated critical point [19, 21] in all $m_{i}$, suggesting that i) an experimental study of these ratios can possibly look for the QCD critical point and ii) it should be accessible to the low energy scan of RHIC at BNL. Indeed, even if one were to be cautious in trusting the numerical precision of the present lattice results, what should be clear is that qualitatively such a non-monotonic behaviour is predicted by the existence of a QCD critical point; the most relevant fact is that the $r_{i j}$ 's used to compute $m_{i}$ had a memory of the lattice result for the QCD critical point in the previous section. To that extent, any model/theory with similar memory of a critical point would lead to a similar non-monotonic behaviour. Moreover, if it is observed at even any other nearby location than those estimates, it would still signal the presence of QCD critical point, albeit somewhat shifted from the points in Fig. 3

In order to confront these results on the baryon number fluctuations with data, one needs to address the issue of neutral baryons - neutrons-which are not easy to detect and are thus missed. It turns out that proton number fluctuations suffice [26]. Since the diverging correlation length at the critical point is linked to the $\sigma$ mode which cannot mix with any isospin modes, and the isospin susceptibility $\chi_{I}$ must be regular there. Assuming protons, neutrons, pions to dominate, Ref [26] showed $\chi_{B}$ to be dominated by proton number fluctuations only. The STAR collaboration has exploited this idea and constructed the ratios $m_{1}$ and $m_{2}$ from net proton distributions [27] in a specific rapidity range $(|y|<0.5)$ and a transverse momentum window $\left(0.4<p_{\mathrm{T}}<0.8 \mathrm{GeV} / c\right)$.

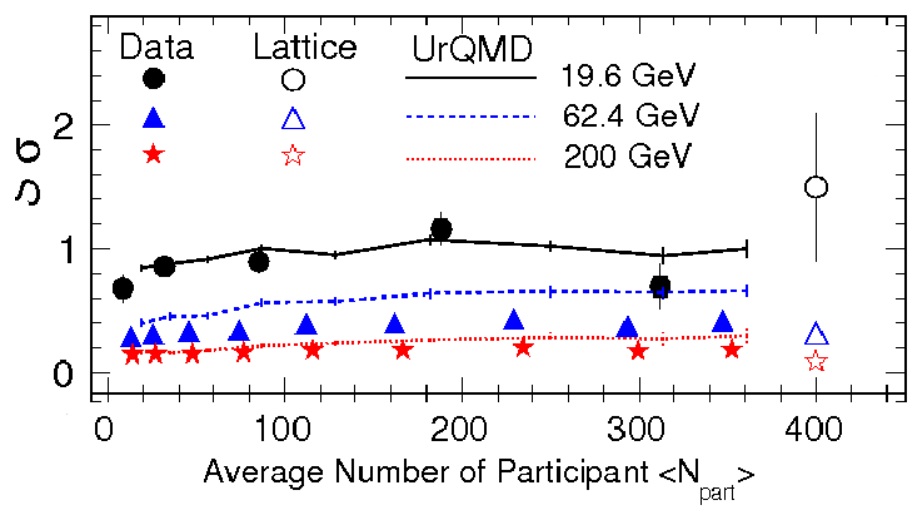

Figure 5. Comparison of the STAR collaboration results with our $m_{1}$ results as a function of number of participants. From Ref. [27].

Figure 5 shows their results for $m_{1}$ ( $S \sigma$ in their notation) against the lattice data [31]. Remarkably, one observes a good agreement with the lattice results. Similar agreement 
is also seen for $m_{2}$. Note that the higher colliding energy, $\sqrt{s}$, corresponds to smaller chemical potential $\mu_{B} / T$. Since higher order terms in the Taylor expansion would clearly be much less significant for such values, the results of [31] are even more reliable. It is very heartening to note that this most direct test of non-perturbative QCD with the experimental data is so good, raising the hope that the results from the RHIC energy scan may be able to locate the critical point this way.

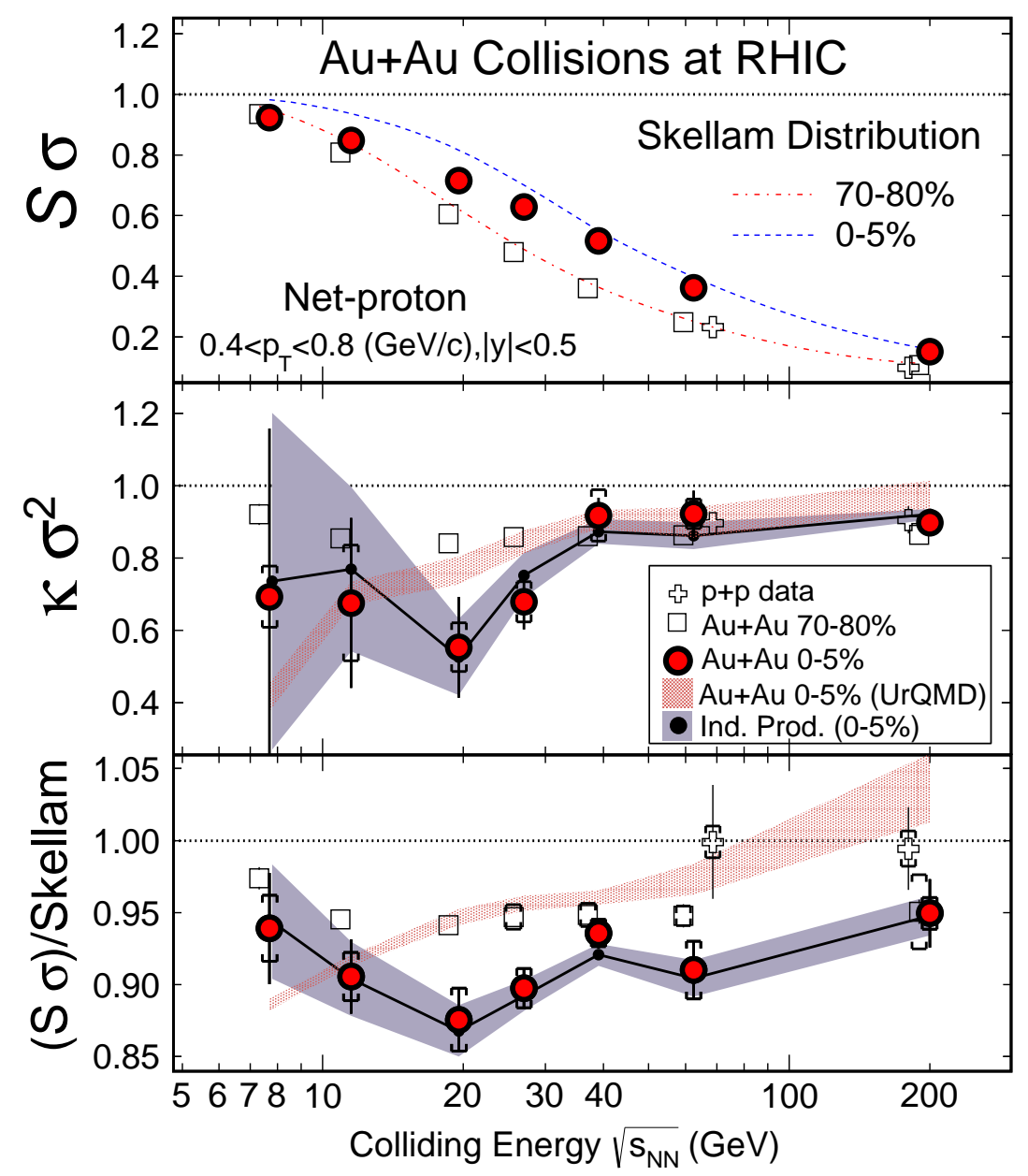

Figure 6. The STAR results for for the equivalent of $m_{1}(S \sigma)$ and $m_{2}\left(\kappa \sigma^{2}\right)$ as a function of the colliding energy. From Ref. [33]. For details, see the text

Figure 6 displays the latest results [33] from the STAR collaboration for the RHIC beam energy scan for $m_{1}\left(S \sigma\right.$ in their notation) and $m_{2}\left(\kappa \sigma^{2}\right.$ in their notation). The latter should be compared with the lattice results [31, 32] for $m_{2}$ in Figure 4 (bottom right panel) for the two finest lattices. As seen in the top panel of Fig. 6, the results for $m_{1}$ do increase with the $\sqrt{s}$, as expected from the lattice results of Fig. 4 and a default hadron resonance gas model with no correlations. The bottom panel therefore exhibits the ratio of the data to the default model to check for deviations. The STAR results for the $0-5 \%$ centrality for Gold-Gold collisions (filled circles) in the two lower panels show a good qualitative and to some extent quantitative agreement with the theoretical predictions 
QCD Critical Point: The Race is On

[31, 32] using the lattice methods. In particular, both show a flat behaviour at large $\sqrt{s}$ and a dip at a lower value. For the lattice results, the dip appears due to the QCD critical point. Whether it is so for the experimental data as well is a million dollar question. One should perhaps check whether the dip is unique at all. The experimentalists checked this by plotting the 70-80\% centrality data (open squares) in the same way. These collisions are expected to be described by simpler nucleon-nucleon like collisions and without any extreme density deposition. One sees no dip for these data. Simulation from the UrQMD model which does not incorporate any QCD critical point does not show a dip even in the 0-5\% centrality data, as seen in Fig. 6. On the other hand, it seems to do a better job for the $70-80 \%$ centrality data. Moreover, the analogue of $m_{1}$ (the top and bottom panels) also show deviations from the expected behaviour, as more vividly seen in the bottom panel of the figure. While these tantalizing results are clearly very exciting, and point towards a possible QCD critical point, more work is still needed on the theoretical and experimental fronts in form of improved precision as well as on dynamic model studies to pin the QCD connection more directly.

For small $z=\mu_{B} / T$, one may invert the relations between $m_{i}$ 's, or similar ratios, and determine in principle the freezeout parameters [31, 34, 35] from the first principles lattice computations as well. Alternatively, one may fit the experimental data to such ratios by varying the scale, the cross-over temperature $T_{c}$, and determine it from the data [36] directly. If the lattice computations were to be really precise, and came with a very reliable estimate of various systematic errors, any of the above approach to extract information would be equally valid, and could even afford a consistency check. What I outlined above in details, is perhaps the most conservative approach which aims to make a comparison of the lattice predictions with the data to discern an imprint of non-perturbative QCD in the experimental data at larger $\sqrt{s}$ and the QCD critical point at the smaller ones. It needed an input scale $T_{c} \simeq 170-175 \mathrm{MeV}$, which appears as a normalization in Fig. 3 , and was needed to relate the dimensionless ratios appearing in the lattice computations to the freeze-out parameters [25].

Recall that the lattice predictions [31, 32] in Figs. 4]and[5]were based on the assumption that the freeze-out curve deduced from hadron abundances represents the true temperature and chemical potential of the fireball when the freeze-out occurs. Even the attempt to determine the freeze-out parameters or the transition temperature $T_{c}$ from the experimental results by using precision lattice results cannot escape from such an assumption. A priori, there is no physical reason for this assumption to be true. Indeed, we do not even have a theoretical proof that thermalization has to be achieved in the heavy ion collisions at all, let alone for the existence of a freeze-out curve. On the other hand, the data based extraction [25] of the freeze-out curve is indeed very impressive and provides the best justification for it.

\section{Summary}

The QCD phase diagram in the $T$ - $\mu_{B}$ plane, Fig. 3, has begun to emerge using first principles lattice approach. Lattice results for $N_{t}=6$ and 8 are first encouraging steps towards continuum limit, suggesting rather small cut-off effects. All lattice results so far suggest a critical point at a small $\mu_{B} / T \sim 1-2$. This maybe very encouraging for the beam energy scan program at RHIC, BNL, which may be able to locate the critical point experimentally. Ratios of nonlinear susceptibilities appear to be smooth on the freeze-out curve at large colliding energy. STAR results on proton number fluctuations in Fig. 5 appear to agree with the lattice QCD predictions [31], making this a unique direct non- 
perturbative test of hot and dense QCD in all such experimental tests so far. Critical point leads to structures in the $m_{i}$, which may be accessible in experiments. So far tell-tale signs of a critical point have emerged in the RHIC experimental results. An interesting question is whether the deviations visible in the RHIC energy scan in Fig. 6 can uniquely be associated with the QCD critical point, as the lattice results [31, 32] in Fig. 4] suggest, or whether mundane explantions can emerge for them. Clearly, a lot of work needs to be done by both theorists and experimentalists, apart from simply improving the precision to resolve this. At any rate, exciting future awaits the beam energy scan at RHIC, and the future programs at FAIR/NICA, as well as all of us interested in it.

\section{Acknowledgements}

It is my great pleasure to acknowledge the wonderful role of my collaborators, Debasish Banerjee, Saumen Datta, Sayantan Sharma, and in particular, Sourendu Gupta from TIFR. My work would not have been possible without the excellent computing facility provided by ILGTI, TIFR, and maintained so well by Ajay Salve and Kapil Ghadiali.

\section{References}

[1] M. Asakawa and K. Yazaki, Nucl. Phys. A504, 668 (1989); A. Barducci, R. Casalbuoni, S. De Curtis, R. Gatto and G. Pettini, Phys. Lett. B 231, 463 (1989).

[2] K. Rajagopal and F. Wilczek, in "At the Frontier of Particle Physics / Handbook of QCD", Vol. 3, M. Shifman, ed., (World Scientific), p2061.

[3] See, e.g., FLAG working group, S. Aoki et al., arXiv:1310.8555

[4] See, e.g., H. J. Rothe, "Lattice Gauge Theries-An Introduction" (World Scientific, 2006) p. 57.

[5] H. Neuberger, Phys. Lett. B 417 (1998) 141; D. B. Kaplan, Phys. Lett. B 288 (1992) 342.

[6] J. Bloch and T. Wettig, Phys. Rev. Lett. 97, 012003 (2006) and Phys. Rev. D76, 114511 (2007).

[7] D. Banerjee, R. V. Gavai and S. Sharma, Phys. Rev. D78, 014506 (2008) and PoS (LATTICE 2008), 177.

[8] R. V. Gavai and S. Sharma, Phys. Rev. D81, 034501 (2010).

[9] R. V. Gavai and S. Sharma, Phys. Lett. B716, 446 (2012).

[10] S. Chandrasekharan, Eur. Phys. J. A 49, 90 (2013).

[11] C. Gattringer, PoS LATTICE 2013, 002 (2013).

[12] Ph. de Forcrand \& O. Philipsen, Nucl. Phys. B642 (2002) 290; M. -P. Lombardo \& M. D’Elia, Phys. Rev. PR D67 (2003) 014505.

[13] K. -F. Liu, Int. J. Mod. Phys. B16 (2002) 2017; S. Kratochvila and Ph. de Forcrand, PoS LAT2005 (2006) 167.

[14] G. Aarts, E. Seiler and I. O. Stamatescu, Phys. Rev. D 81 (2010) 054508 and references therein.

[15] Z. Fodor \& S. Katz, JHEP 0203 (2002) 014; JHEP 0404, 050 (2004).

[16] R. V. Gavai and S. Gupta, Phys. Rev. D68, 034506 (2003).

[17] C. Allton et al.,Phys. Rev. D68, 014507 (2003).

[18] R. V. Gavai and S. Gupta, Phys. Rev. D71, 114014 (2005).

[19] R. V. Gavai and S. Gupta, Phys. Rev. D78, 114503 (2008).

[20] R. V. Gavai and S. Sharma, Nucl. Phys. A 862-863CF, 355 (2011); Phys. Rev. D 85, 054508 (2012).

[21] S. Datta, R. V. Gavai and S. Gupta, PoS (LATTICE 2013), 202 (2013).

[22] S. Gottlieb et al, Phys. Rev. Lett. 59, 1513 (1987).

[23] J. D. Bjorken, Phys. Rev. D 27, 140 (1983). 
[24] For an introductory review, see, R. S. Bhalerao and R. V. Gavai, in "Physics at the Large Hadron Collider", INSA Platinum Jubilee issue, (Springer, 2009), Eds. A. Datta, B. Mukhopadhyaya and A. Raychaudhari, p. 105.; For latest results, Proceedings of "Quark Matter 2012", Nucl. Phys. A904-905(2013).

[25] A. Andronic, P. Braun-Munzinger and J. Stachel, Phys. Lett. , B 673 (2009) 142; H. Oeschler, J. Cleymans, K. Redlich, S. Wheaton, arXiv:0910.2128

[26] Y. Hatta and M. A. Stephenov, Phys. Rev. Lett. 91 (2003)102003.

[27] M. M. Agrawal et al., Phys. Rev. Lett. 105 (2010) 022302 [arXiv : 1004.4959].

[28] M. A. Stephanov, K. Rajagopal and E. V. Shuryak, Phys. Rev. Lett. 81, 4816 (1998); Phys. Rev. D 60, 114028 (1999).

[29] M. A. Stephanov, Phys. Rev. Lett. 102, 032301 (2009); C. Athanasiou, K. Rajagopal and M. A. Stephanov, Phys. Rev. D 82, 074008 (2010).

[30] S. Gupta, PoS (CPOD2009) 025 (2009).

[31] R. V. Gavai and S. Gupta, Phys. Lett. B 696, 459 (2011)

[32] S. Datta, R. V. Gavai and S. Gupta, Poster presented at the "Quark Matter 2012", Washington, USA, 13-18 August 2012.

[33] L. Adamczyk et al. [STAR Collaboration], Phys. Rev. Lett. 112, 032302 (2014).

[34] A. Bazavov, H. T. Ding, P. Hegde, O. Kaczmarek, F. Karsch, E. Laermann, S. Mukherjee and P. Petreczky et al., Phys. Rev. Lett. 109, 192302 (2012).

[35] S. Borsanyi, Z. Fodor, S. D. Katz, S. Krieg, C. Ratti and K. K. Szabo, arXiv:1403.4576 [heplat]; arXiv:1311.7397[hep-lat].

[36] S. Gupta, X. Luo, B. Mohanty, H. G. Ritter and N. Xu, Science 332, 1525 (2011) 